OPEN ACCESS

Edited by:

Brian Keith McFarlin,

University of North Texas,

United States

Reviewed by:

Asghar Abbasi,

Los Angeles Biomedical Research Institute, United States

Y. Gul Ozkaya,

Akdeniz University, Turkey

*Correspondence:

Brandt D. Pence

bdpence@memphis.edu

Specialty section:

This article was submitted to

Exercise Physiology,

a section of the journal

Frontiers in Physiology

Received: 12 October 2017 Accepted: 19 December 2017

Published: 05 January 2018

Citation:

Pence BD, Ryerson MR,

Bravo Cruz AG, Woods JA and

Shisler JL (2018) Voluntary Wheel

Running Does Not Alter Mortality to or Immunogenicity of Vaccinia Virus in

Mice: A Pilot Study

Front. Physiol. 8:1123.

doi: 10.3389/fphys.2017.01123

\section{Voluntary Wheel Running Does Not Alter Mortality to or Immunogenicity of Vaccinia Virus in Mice: A Pilot Study}

\author{
Brandt D. Pence ${ }^{1,2,3,4 *}$, Melissa R. Ryerson ${ }^{5}$, Ariana G. Bravo Cruz ${ }^{5}$, Jeffrey A. Woods ${ }^{3,4}$ \\ and Joanna L. Shisler ${ }^{5}$ \\ ${ }^{1}$ School of Health Studies, University of Memphis, Memphis, TN, United States, ${ }^{2}$ Center for Nutraceutical and Dietary \\ Supplement Research, University of Memphis, Memphis, TN, United States, ${ }^{3}$ Department of Kinesiology and Community \\ Health, University of Illinois Urbana-Champaign, Urbana, IL, United States, ${ }^{4}$ Integrative Immunology and Behavior Program, \\ University of Illinois Urbana-Champaign, Urbana, IL, United States, ${ }^{5}$ Department of Microbiology, University of Illinois \\ Urbana-Champaign, Urbana, IL, United States
}

Exercise has been shown to improve immune responses to viral infections and vaccines in several mouse models. However, previous pathogen studies have primarily used infections limited to the respiratory tract. Additionally, previous studies have utilized forced treadmill exercise paradigms, and voluntary wheel running (VWR) has been shown to have differential effects on the immune system in non-infection models. We examined whether WWR could improve morbidity and mortality to a 50\% lethal dose of vaccinia virus (VACV), a systemic pathogen commonly used to examine immune responses. Additionally, we examined whether VWR could improve antibody response to a replication-deficient strain of VACV, mimicking a vaccination. Male C57BI/6J mice underwent 8 weeks of WWR or remained sedentary, then were infected intranasally with $10^{5}$ PFU VACV strain WR and followed 14 days for weight loss. Mice in the vaccination study ran or were sedentary for 8 weeks, then were given $10^{6}$ PFU of replication-deficient VACV strain MVA intraperitoneally. Blood was collected at 1, 2, and 4 weeks post-inoculation, and anti-VACV IgG titer was determined by ELISA. WWR did not improve mortality due to VACV infection $(p=0.26)$, although fewer VWR mice $(4 / 10)$ died compared to sedentary (SED, 6/10). WWR did not prevent body weight loss due to infection compared to SED $(p=0.20)$, although WWR mice loss slightly less weight compared to SED through the first 6 days post-infection. Food intake was significantly reduced in SED post-infection compared to WWR $(p=0.05)$. WWR mice developed a greater IgG antibody response, although this was not significant $(p=0.22)$. In summary, WWR did not protect against mortality to VACV or prevent infection-induced weight loss, and WWR did not enhance antibody responses. However, there were non-significant trends toward WWR-related improvements in these outcomes, and post-infection food intake was improved by VWR.

Keywords: exercise, voluntary wheel running, infection, virus, vaccinia virus, mortality 


\section{INTRODUCTION}

Interventions which improve immune function and/or vaccine response, including exercise, are of critical importance in the field of public health. This is particularly true in populations at risk of poor immune responses, including individuals with obesity and individuals undergoing normal aging (Milner and Beck, 2012; Montecino-Rodriguez et al., 2013). However, prior to research in at-risk populations, the safety and efficacy of the combination of viral infection and exercise must be studied in healthy populations. These types of studies have been performed previously using influenza virus (Lowder et al., 2005, 2006; Sim et al., 2009) and herpes simplex virus (HSV)-1 (Kohut et al., 2001).

However, both influenza virus and HSV-1 are infections limited to the airway in mice. To date, no studies have been performed using systemic infections. Vaccinia virus (VACV) is a poxvirus which can be administered by a variety of routes and causes a systemic infection in mice (Hutchens et al., 2008). Poxviruses such as VACV are highly important from a public health perspective for several reasons. Historically, smallpox (variola virus) is potentially the most devastating human disease, with mortality rates of up to $30 \%$ prior to its eradication in 1980 (Nafziger, 2005). Despite the eradication of smallpox, there still exists a threat of the emergence of the disease or related poxviruses such as monkeypox, due either to natural mutations (in the case of monkeypox) or due to intentional release as biological weapons. Should such instances occur, vaccination with less-deadly poxviruses such as VACV is likely to be reimplemented.

Additionally, poxviruses such as wild-type and mutated VACV strains are commonly being used as vectors for vaccines for other pathogens (Lousberg et al., 2011) as well as for noninfectious diseases such as cancer (Kim and Gulley, 2012). VACV is highly genetically tractable, a major advantage in molecular biology research. VACV is a double-stranded DNA virus which encodes approximately 250 proteins, many with functions which modulate the host's immune response directly (Lousberg et al., 2011).

We undertook a small pilot study to examine the impact of exercise training on the immune response to VACV, using both a live viral infection model and an attenuated vaccine strain model in mice. Our goals were to establish whether exercise training was efficacious at improving immune responses to VACV. A secondary goal was to determine if moderate exercise was safe in the event of VACV exposure by infection or deliberate inoculation. We hypothesized that exercise training in mice would reduce viral infection-mediated mortality and improve the antibody response to the vaccine strain. A thorough understanding of the relationship between exercise training and the immune response to vaccinia virus could be important in the future both for understanding the basic mechanisms by which exercise alters immune function, as well as for ensuring the safety and efficacy of vaccinations with VACV-derived viral strains in the event they are reintroduced.

\section{METHODS}

\section{Mice}

Six week old male C57Bl/6J mice $(N=38)$ were acquired from Jackson Laboratories (Bar Harbor, ME) and acclimated for 1 week in our facility prior to onset of the exercise protocol. All mice received ad libitum access to a rodent chow diet and water during the course of the study. Mice were housed on a 12-h light-dark cycle with lights on at 0800 each day. All experimental procedures were approved by the Institutional Animal Care and Use Committee at the University of Illinois Urbana-Champaign, and carried out in an AAALAC-accredited facility under biosafety level 2 conditions.

\section{Exercise}

Mice exercised or remained sedentary for 8 weeks prior to VACV infection. The wheel running group (Wh) was given ad libitum access to stainless steel running wheels both for the 8 weeks prior to inoculation and for the period between inoculation and mortality/euthanasia. Sedentary mice (Sed) remained in their home cages for the duration of the study. All mice underwent similar handling.

\section{Viruses}

Mice were infected with VACV strain Western Reserve (WR) for determination of morbidity and mortality responses. WR was cultured in BHK-1 cells (ATCC, Manassas, VA), titrated by plaque assay using standard techniques, and purified by ultracentrifugation. Mice were inoculated under anesthesia by inhaled isoflurane with $10^{5} \mathrm{PFU} \mathrm{WR}$ in $20 \mu \mathrm{l} \mathrm{PBS}$, given intranasally as $10 \mu \mathrm{l}$ per nostril. This dose of WR was verified to cause approximately 50\% mortality in sedentary mice in a preliminary experiment prior to the study (data not shown). For antibody responses, mice were inoculated by intraperitoneal injection with $10^{6} \mathrm{PFU}$ of replicationdeficient Modified Vaccinia Ankara (MVA) virus in $100 \mu \mathrm{l}$ PBS. MVA was cultured in CEF cells (ATCC), titrated by plaque assay, and purified by ultracentrifugation prior to inoculation.

\section{Morbidity and Mortality}

Morbidity was measured by examining reductions in daily food intake and body weights for 6 days post-infection with WR, as significant mortality took effect beginning on day 7 postinfection. Body weight was monitored by daily weighing with a digital scale. Food intake was measured by food disappearance by subtracting the current day's food weight from the previous day's food weight. Typical subjective scoring measures used to assess morbidity could not be used in this study due to the need to manipulate mice only under a biosafety cabinet, which precluded blinding of investigators to the presence or absence of a running wheel. For mortality, mice were euthanized once they reached a weight loss of $30 \%$ of pre-infection weight, the point at which WR-infected mice have been shown to fail to recover from viral infection (Bartlett et al., 2002). A small number of mice died 
naturally from WR infection prior to reaching this body weight cutoff.

\section{Antibody Responses}

Prior to MVA injection and at 1,2, and 4 weeks post-inoculation, mice were anesthetized by inhalation with isoflurane in a flow rate of $2-3 \mathrm{~L} \cdot \mathrm{min}^{-1}$ oxygen. Blood $(200 \mu \mathrm{l})$ was collected from the retro-orbital sinus with a glass Pasteur pipette into heparinized microcentrifuge tubes, then centrifuged at 1,500 $\times$ $g$ for $15 \mathrm{~min}$ to separate plasma. Plasma was stored at $-80^{\circ} \mathrm{C}$.

Anti-vaccinia IgG response was determined by enzyme-linked immunosorbant assay. Briefly, 96-well plates (Corning, Corning, NY) were coated with PBS containing $1 \mu \mathrm{g}$ whole cell lysate total protein from MVA-infected CEF cells or mock-infected CEF cells (as non-specific binding controls) and allowed to attach overnight at $4^{\circ} \mathrm{C}$. Binding sites were then blocked for $1 \mathrm{~h}$ with $1 \%$ bovine serum albumin at room temperature, followed by addition of plasma diluted 1:100 in PBS. After a $2 \mathrm{~h}$ incubation at room temperature, plates were washed and $50 \mu \mathrm{l}$ of 1:800 rabbit anti-mouse IgG-HRP (Life Techonologies, Frederick, MD) was added to each well. Plates were incubated for $1 \mathrm{~h}$ at room temperature and washed, followed by incubation for $20 \mathrm{~min}$ in 50 $\mu l$ of TMB substrate reagent (1:1 mixture of TMB and hydrogen peroxide, BD Biosciences, San Jose, CA) and read at $405 \mathrm{~nm}$ on a spectrophotometric plate reader (BioTek, Winooski, VT).

Plasma IgG optical density (O.D.) from binding to mock infected CEF lysates was subtracted from that to MVA-infected $\mathrm{CEF}$ lysates to correct for non-specific antibody binding. Plasma IgG values were expressed as change in O.D. from the preinoculation measure.

\section{Data Analysis}

Data were analyzed with GraphPad Prism 5 (La Jolla, CA) unless otherwise noted. Mortality was assessed by the Mantel-Cox test, comparing survival portions of Wh vs. Sed. Body weight and food intake data during infection with WR were analyzed by repeated measures analysis of variance (RM-ANOVA), with Bonferroni post-hoc correction in the event of a significant main effect or interaction. Antibody response data were also assessed with RM-ANOVA using the same methods. For proportional tests for threshold antibody responses at week 4 post-inoculation, a Pearson's chi-square test was performed using R v. 3.2.1 (R Foundation, Vienna, AUS). For post-hoc power analyses using mortality and antibody response results, power was calculated using the "pwr" package in R v. 3.2.1. Significance was set at $p \leq 0.05$.

\section{RESULTS}

\section{Morbidity and Mortality}

No significant interactions were recorded for morbidity and mortality metrics, therefore we report main effects of exercise in this section. Wh did not significantly improve mortality rates due to infection with WR, although mice in the Wh group did survive at a higher rate $(60 \%)$ than those in the Sed group (40\%, $p=0.26$, Figure 1A). Additionally, body weight did not differ during infection with WR, although again the Sed group
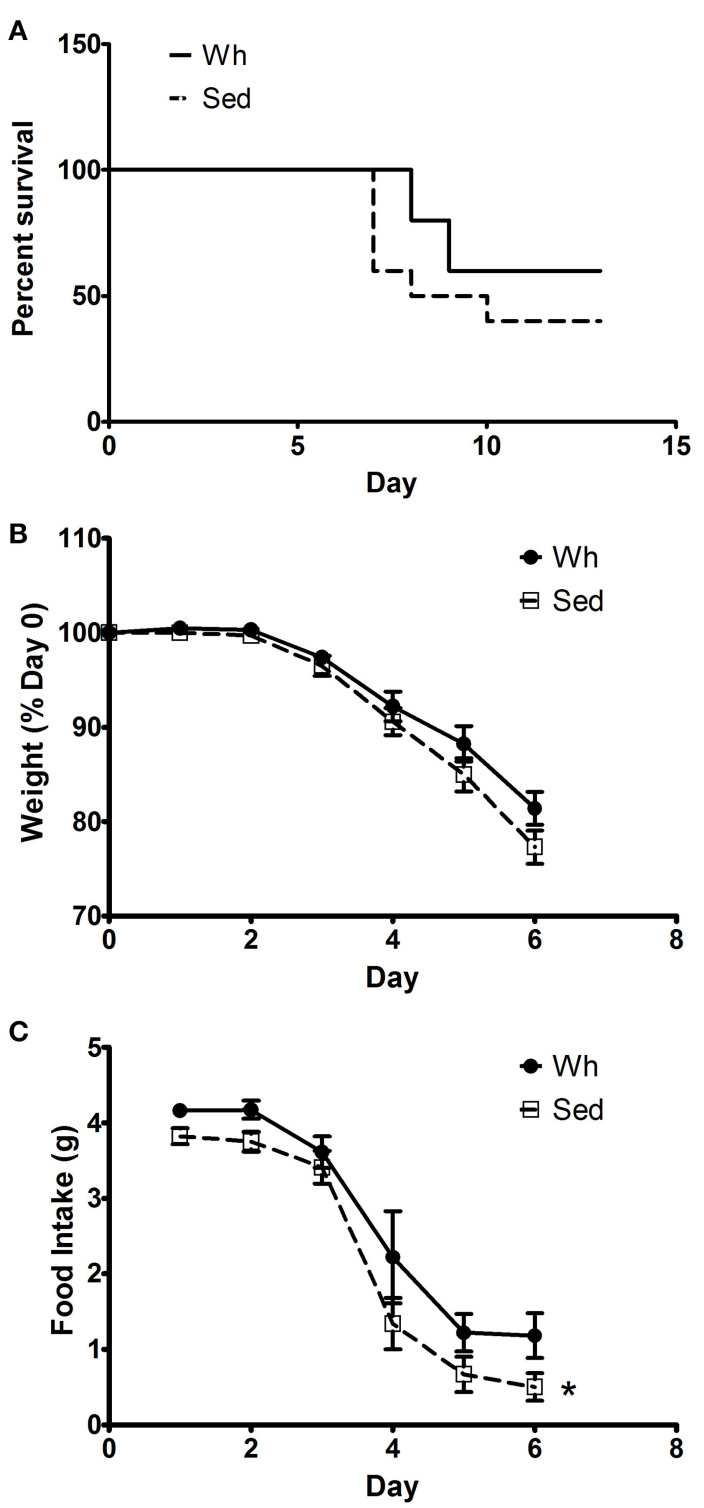

FIGURE 1 | Morbidity and mortality response to infection with VACV strain WR. (A) Percentage survival by day post-infection. (B) Body weight as a percentage of pre-infection weight. (C) Daily food intake post-infection. *Main effect of activity $(\rho<0.05)$. Wh, wheel mice. Sed, sedentary mice. $N=$ 10/group.

lost more $4 \%$ weight than those in the Wh group $(p=0.20$, Figure 1B). Promisingly, food intake was significantly reduced in the Sed group compared to the Wh group ( $p=0.05$, Figure 1C), suggestive of a Wh effect on morbidity.

\section{Antibody Responses}

We examined the kinetic antibody response to intraperitoneal injection of MVA by ELISA (Figure 2A). Inoculation of MVA induced a significant increase in anti-VACV IgG in both groups (overall time main effect $p<0.001$ ). Although the Wh group had greater induction of IgG response, especially at later time 

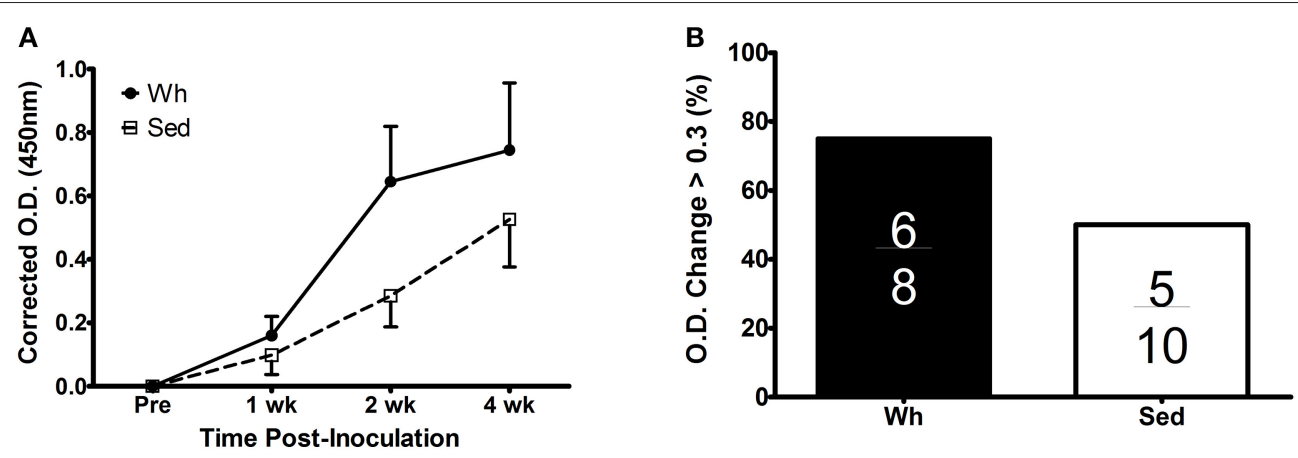

FIGURE 2 | Antibody response to inoculation with VACV strain MVA. (A) Plasma anti-VACV IgG at pre-inoculation and at 1, 2, and 4 weeks post-inoculation, corrected for non-specific binding and expressed as difference from baseline (B) Proportion of mice with O.D. increase of $>0.3$ from pre-inoculation to 4 weeks post-inoculation. Inset fractions indicate mice meeting the change criteria out of total mice in the group. Wh, wheel mice $(N=8)$. Sed, sedentary mice $(N=10)$. O.D., optical density.

points (i.e., week 2 and week 4 post inoculation), the main effect of exercise was not significant $(p=0.22)$, likely due to the high variability of antibody response. We also examined the proportion of responses in which O.D. increased by $>0.3$ from pre-inoculation to week 4 post-inoculation (Figure 2B). Although 6/8 Wh mice met this standard, compared to only $5 / 10$ Sed mice, this proportion was not significantly different by Pearson's chi-squared test $(p=0.28)$.

\section{DISCUSSION}

Exercise has long been known to have immunomodulatory properties which alter the response of the host to viral infections. Early work by Nieman and colleagues demonstrated that higher levels of exercise training reduced symptoms of upper respiratory tract infection (Nieman et al., 1989), and that exercise training interventions could similarly reduce the rate of reported symptoms (Nieman et al., 1990, 1993). Because human studies are generally limited to symptom reports for ethical reasons, and because reported symptom are not necessarily related to actual pathogen exposure (Spence et al., 2007), experimental studies using viral infections in rodent models were undertaken by several investigators in order to directly study the effects of exercise on viral infections.

One of the earliest studies along these lines (Kohut et al., 2001) demonstrated that 8 weeks of moderate treadmill training enhanced antiviral antibody responses and production of Th1-associated cytokines in aged mice in response to HSV1. Additionally, a four-day moderate exercise program (30 min $\cdot$ day $^{-1}$ ) given post-infection (but prior to symptom onset) reduced mortality to influenza virus in mice (Lowder et al., 2005), while a 3.5 month chronic exercise training program showed similar benefits in mice exposed to influenza virus infection (Bartlett et al., 2002).

The above studies share several characteristics, most notably that (i) exercise was conducted using forced treadmill running, and (ii) viral infections were limited to the respiratory tract. To date, we are aware of no research which has examined the impact of VWR on the response to viral infection in mice. This is important, as previous research has demonstrated that VWR and forced treadmill running can modulate the immune response in different (and indeed opposite) ways (Cook et al., 2013). Therefore, we chose to use VWR as our exercise model for this pilot study. Additionally, we chose to use VACV, a virus that is not limited to the respiratory tract, as a more general model for viral infections in this study.

In short, we found that VWR in mice did not alter the antibody response to the attenuated MVA strain of VACV, nor did it change the morbidity or mortality response to the pathogenic WR strain. However, we noted general trends for improvements with exercise, both in reducing morbidity and mortality to WR and in enhancing anti-VACV IgG responses.

It appears that the variability in the responses in these mice makes it difficult to confirm differences in small sample sizes, such as the 8-10 per group used in this study. Sensitivity analyses suggest that 60 mice per group would be necessary to conclude that exercise is protective against mortality from WR infection, and that more than 120 mice per group would be necessary to conclude that antibody responses are different at 4 weeks post-MVA inoculation. A study of this magnitude is impractical and would have low benefit-to-cost ratio, given the variability and small effect sizes seen here. Effectively, the use of so many additional mice to support what is likely a small (and potentially not physiologically-relevant) exercise effect in this case is, in our view, unethical in the light of significant burden to the additional mice for little gain.

It is likely that young healthy mice, such as those used in this study, have sufficiently strong immune responses such that exercise is not enough to induce a large improvement. This exercise paradigm may be more efficacious in models with impaired immune responses to viral infections, such as aging (Montecino-Rodriguez et al., 2013) or obesity (Milner and Beck, 2012). Exercise has recently been shown to enhance immunity to influenza infection in obese mice (Warren et al., 2015), and a similar effect is possible using this VACV infection model.

Despite the non-significant findings in this study, our results do demonstrate relatively convincingly that moderate exercise training is unlikely to either decrease the safety and efficacy of a VACV-based vaccination or to enhance the pathogenicity of a poxviral infection. These outcomes are important, given the 
increasing use of MVA and similar attenuated VACV strains in experimental vaccines (Hutchens et al., 2008; Lousberg et al., 2011).

In conclusion, moderate exercise training by voluntary wheel running did not alter morbidity or mortality to infection with pathogenic VACV strain WR, and did not alter antibody responses to a vaccination challenge with attenuated VACV strain MVA. Small effect sizes and high variability obscured the general trend toward improved responses with exercise in this study, and future research in models with impaired immune responses may hold more promise.

\section{REFERENCES}

Bartlett, N., Symons, J. A., Tscharke, D. C., and Smith, G. L. (2002). The vaccinia virus N1L protein is an intracellular homodimer that promotes virulence. $J$. Gen. Virol. 83, 1965-1976. doi: 10.1099/0022-1317-83-8-1965

Cook, M. D., Martin, S. A., Williams, C., Whitlock, K., Wallig, M. A., Pence, B. D., et al. (2013). Forced treadmill exercise training exacerbates inflammation and causes mortality while voluntary wheel training is protective in a mouse model of colitis. Brain Behav. Immun. 33, 46-56. doi: 10.1016/j.bbi.2013. 05.005

Hutchens, M., Luker, K. E., Sottile, P., Sonstein, J., Lukacs, N. W., Nunez, G., et al. (2008). TLR3 increases disease morbidity and mortality from vaccinia infection. J. Immunol. 180, 483-491. doi: 10.4049/jimmunol.180.1.483

Kim, J. W., and Gulley, J. L. (2012). Poxviral vectors for cancer immunotherapy. Expert Opin. Biol. Ther. 12, 463-478. doi: 10.1517/14712598.2012.668516

Kohut, M. L., Boehm, G. W., and Moynihan, J. A. (2001). Moderate exercise is associated with enhanced antigen-specific cytokine, but not IgM antibody production in aged mice. Mech. Ageing Dev. 122, 1135-1150. doi: 10.1016/S0047-6374(01)00255-X

Lousberg, E. L., Diener, K. R., Brown, M. P., and Hayball, J. D. (2011). Innate immune recognition of poxviral vaccine vectors. Expert Rev. Vaccines 10, 1435-1449. doi: 10.1586/erv.11.121

Lowder, T., Padgett, D. A., and Woods, J. A. (2005). Moderate exercise protects mice from death due to influenza virus. Brain Behav. Immun. 19, 377-380. doi: 10.1016/j.bbi.2005.04.002

Lowder, T., Padgett, D. A., and Woods, J. A. (2006). Moderate exercise early after influenza virus infection reduces the Th1 inflammatory response in lungs of mice. Exerc. Immunol. Rev. 12, 97-111.

Milner, J. J., and Beck, M. A. (2012). The impact of obesity on the immune response to infection. Proc. Nutr. Soc. 71, 298-306. doi: 10.1017/S0029665112000158

Montecino-Rodriguez, E., Berent-Maoz, B., and Dorshkind, K. (2013). Causes, consequences, and reversal of immune system aging. J. Clin. Invest. 123, 958-965. doi: 10.1172/JCI64096

Nafziger, S. D. (2005). Smallpox. Crit. Care Clin. 21, 739-746. doi: $10.1016 /$ j.ccc.2005.06.004

\section{AUTHOR CONTRIBUTIONS}

BP conceived the study. BP, JW, and JS designed the study. BP, $\mathrm{MR}$, and $\mathrm{AB}$ collected data. $\mathrm{BP}$ analyzed and interpreted the data and drafted the manuscript. $\mathrm{BP}, \mathrm{MR}, \mathrm{AB}, \mathrm{JW}$, and JS revised the manuscript and approved the final version.

\section{FUNDING}

Funding for this project was provided by an ACSM Research Endowment grant to BP.

Nieman, D. C., Henson, D. A., Gusewitch, G., Warren, B. J., Dotson, R. C., Butterworth, D. E., et al. (1993). Physical activity and immune function in elderly women. Med. Sci. Sports Exerc. 25, 823-831. doi: 10.1249/00005768-199307000-00011

Nieman, D. C., Johanssen, L. M., and Lee, J. W. (1989). Infectious episodes in runners before and after a roadrace. J. Sports Med. Phys. Fitness 29, 289-296.

Nieman, D. C., Nehlsen-Cannarella, S. L., Markoff, P. A., Balk-Lamberton, A. J., Yang, H., Chritton, D. B., et al. (1990). The effects of moderate exercise training on natural killer cells and acute upper respiratory tract infections. Int. J. Sports Med. 11, 467-473. doi: 10.1055/s-2007-1024839

Sim, Y. J., Yu, S., Yoon, K. J., Loiacono, C. M., and Kohut, M. L. (2009). Chronic exercise reduces illness severity, decreases viral load, and results in greater antiinflammatory effects than acute exercise during influenza infection. J. Infect. Dis. 200, 1434-1442. doi: 10.1086/606014

Spence, L., Brown, W. J., Pyne, D. B., Nissen, M. D., Sloots, T. P., McCormack, J. G., et al. (2007). Incidence, etiology, and symptomatology of upper respiratory illness in elite athletes. Med. Sci. Sports Exerc. 39, 577-586. doi: 10.1249/mss.0b013e31802e851a

Warren, K. J., Olson, M. M., Thompson, N. J., Cahill, M. L., Wyatt, T. A., Yoon, K. J., et al. (2015). Exercise improves host response to influenza viral infection in obese and non-obese mice through different mechanisms. PLoS ONE 10:e0129713. doi: 10.1371/journal.pone.0129713

Conflict of Interest Statement: The authors declare that the research was conducted in the absence of any commercial or financial relationships that could be construed as a potential conflict of interest.

Copyright (c) 2018 Pence, Ryerson, Bravo Cruz, Woods and Shisler. This is an openaccess article distributed under the terms of the Creative Commons Attribution License (CC BY). The use, distribution or reproduction in other forums is permitted, provided the original author(s) or licensor are credited and that the original publication in this journal is cited, in accordance with accepted academic practice. No use, distribution or reproduction is permitted which does not comply with these terms. 